\title{
Kinetics and chemical analysis of photoinduced interdiffusion in nanolayered $\mathrm{Se} / \mathrm{As}_{2} \mathrm{~S}_{3}$ films
}

\author{
K. V. Adarsh, ${ }^{1, a)}$ Ramakanta Naik, ${ }^{1}$ K. S. Sangunni, ${ }^{1, b)}$ S. Kokenyesi, ${ }^{2}$ H. Jain, ${ }^{3}$ and \\ Alfred C. Miller ${ }^{4}$ \\ ${ }^{1}$ Department of Physics, Indian Institute of Science, Bangalore 560 012, India \\ ${ }^{2}$ Department of Experimental Physics, University of Debrecen, Bem Terr, Debrecen 4026, Hungary \\ ${ }^{3}$ Department of Materials Science and Engineering, Lehigh University, Bethlehem, Pennsylvania 18015, USA \\ ${ }^{4}$ Zettlemoyer Centre for Surface Studies, Lehigh University, Bethlehem, Pennsylvania 18015, USA
}

(Received 26 March 2008; accepted 22 June 2008; published online 2 September 2008)

\begin{abstract}
We have studied the kinetics of photoinduced effects in nanolayered $\mathrm{Se} / \mathrm{As}_{2} \mathrm{~S}_{3}$ film by in situ optical absorption measurements, which reveal that photodarkening in these films is followed by photoinduced diffusion. An increase in disorder during photodarkening and its subsequent decrease during photoinduced diffusion were also observed. The observation of photodarkening of Se at room temperature when confined between $\mathrm{As}_{2} \mathrm{~S}_{3}$ layers suggests that the glass transition temperature of Se shifts to higher energy. The analysis shows that the atoms which take part in photodarkening play a vital role in photoinduced diffusion. The x-ray photoelectron spectroscopy measurements show the atomic movements during photoinduced diffusion. It also shows that some of the As-S bonds are converted into As-Se bonds. Since it is energetically difficult to break an As-S bond to form an As-Se bond, we assume that the new bond formations are taking place by the bond rearrangement mechanism. (C) 2008 American Institute of Physics. [DOI: 10.1063/1.2973460]
\end{abstract}

\section{INTRODUCTION}

Recently chalcogenide glass multilayers were prepared with high quality nanomodulation, which demonstrated their potential for tailoring the optical properties. ${ }^{1-3}$ These amorphous nanolayered chalcogenide (ANC) structures are similar to the crystalline superlattices yet distinct from ideal crystalline superlattices produced by molecular beam epitaxy. The ANCs can be considered as well-correlated layers with good periodicity and smooth interface. The surface analysis with an atomic force microscope showed that their surface roughness is $0.5-1 \mathrm{~nm}$. They are attractive because of the prominent photoinduced effects, similar to those exhibited by uniform thin films. ${ }^{2,4}$ For example, photoinduced diffusion in short period multilayer systems is important because of its potential applications in holographic recording and fabrication of phase gratings. ${ }^{1}$ In spite of its practical usefulness, the mechanism of photoinduced diffusion is not properly understood.

Silver metal photoinduced diffusion in chalcogenide glasses is well known for many decades. ${ }^{5}$ By comparison, photoinduced diffusion has been observed only recently in amorphous $\mathrm{Se} / \mathrm{As}_{2} \mathrm{~S}_{3}$ and similar multilayers. The diffusional intermixing affects several parameters of the multilayer structure, such as optical absorption edge, layer thickness, photoluminescence intensity, electrical conductivity, and photoconductivity. ${ }^{2,3,6}$ The experiments indicate that the light- and thermostimulated diffusion effects are rather similar. The changes in these properties are explained based on the formation of ternary solid solution due to interdiffu-

\footnotetext{
a) Also at Department of Materials Science and Engineering, Lehigh University, Bethlehem, Pennsylvania18015.

b) Author to whom correspondence should be addressed. Electronic mail: sangu@physics.iisc.ernet.in.
}

sion. However, the process by which Se diffuses into $\mathrm{As}_{2} \mathrm{~S}_{3}$ is still unclear. Our previous studies revealed that the bandgap and Tauc parameter increase after photoinduced diffusion. ${ }^{2,3}$ It was assumed that diffusion takes place mainly in strained sites where wrong bonds are close to $\mathrm{Se}_{2}^{0}$ sites (the subscripts and superscripts denote the coordination number and charge state, respectively). ${ }^{3}$ Diffusion takes place through the reaction of $\mathrm{Se}_{2}^{0}$ with the wrong $\mathrm{S}-\mathrm{S}\left(\mathrm{S}_{2}^{0}\right)$ and As-As bonds. The $\mathrm{Se}_{2}^{0} / \mathrm{S}_{2}^{0}$ defects are known as annihilating defects (ADs). During light irradiation, the decrease in photoinduced viscosity leads to a mechanical stress gradient and thus enables the directed motion of $\mathrm{ADs}$. $\mathrm{Se}_{2}^{0}$ reacts with $\mathrm{S}_{2}^{0}$, forming $\mathrm{S}-\mathrm{Se}$ bonds and converting As-As to As-Se bond. A cooperative annihilation of ADs (because of mechanical stresses in a continuous glass network and strong electron-phonon interaction in amorphous chalocogenides) results in the formation of As-Se, S-Se, and As-S bonds. Note that all previous measurements were performed ex situ, i.e., a film exposed under light irradiation during the measurement was never studied. In situ changes in the transmission spectra were measured but at a fixed wavelength. Since the measurements were done on a single wavelength, the kinetics of the variation of optical bandgap and Tauc parameter could not be obtained. In short, information has been missing about the transient or metastable changes in the multilayer structure during photoinduced diffusion. In this paper, we report in situ changes in transmission spectra recorded over the wavelength range $\lambda=400-1000 \mathrm{~nm}$ and also at fixed wavelengths to understand the changes in absorption coefficient, optical bandgap, and Tauc parameter during photoinduced diffusion.

Since the optical absorption measurements will not directly give any visualization of the mass transport of atoms during photoinduced diffusion, we used x-ray photoelectron 
spectroscopy (XPS) to study the atomic movements. XPS is also used to analyze the new bonds formed between the components due to interdiffusion. It is a useful surface analytical technique to study the chemical state and local environment of an atom. ${ }^{7,8}$ The chemical bonding is often realized through correlation with chemical shifts in XPS binding energies of the corresponding elements. When the x-ray photon impinges on a sample, photoionization takes place and an electron is expelled from the sample with a certain kinetic energy. The energy of the photoelectrons leaving the sample gives a spectrum with a series of peaks. The binding energies of the peaks are characteristic of each element. Since XPS is a surface analytical technique, most of spectra will come from the top 70-100 $\AA$ layer. To study the atomic movement we irradiated the sample inside the XPS chamber and measured the spectra. The scanning speed of the XPS peaks is very slow and hence it is not advisable to use an in situ measurement. Therefore, we irradiated the sample for a desired time inside the XPS chamber and the XPS spectra were recorded after switching off the laser. The disadvantage of this method is that the transient effects are not visible and nevertheless gives an idea of the atomic movements which are permanent ones. For these experiments, the samples were prepared with Se as the top layer and $\mathrm{As}_{2} \mathrm{~S}_{3}$ as the bottom layer. The thickness of the top Se layer was optimized in such a way that there will not be any $\mathrm{As}_{2} \mathrm{~S}_{3}$ signal during the measurement of an as prepared film. During illumination, $\mathrm{As}_{2} \mathrm{~S}_{3}$ will diffuse into the Se and this atomic movement will give the particular XPS signal of As and $\mathrm{S}$ atoms. These experiments will show the direct observation of the atomic movements. Even though there are many publications about the Se diffusion in $\mathrm{As}_{2} \mathrm{~S}_{3}$, there is no experimental proof for the atomic movements. With the help of this experiment, we were able to show the atomic movement of As, $\mathrm{S}$ inside the Se matrix, and also confirm that the changes in optical and electrical properties of $\mathrm{Se} / \mathrm{As}_{2} \mathrm{~S}_{3}$ nanolayered films during illumination are due to the formation of the solid solution of $\mathrm{Se}-\mathrm{As}_{2} \mathrm{~S}_{3}$.

\section{EXPERIMENTAL PROCEDURES}

Nanolayered $\mathrm{Se} / \mathrm{As}_{2} \mathrm{~S}_{3}$ films were prepared by cyclic thermal evaporation of bulk Se and $\mathrm{As}_{2} \mathrm{~S}_{3}$. Deposition rates were $2-10 \mathrm{~nm} / \mathrm{s}$ in a vacuum of $5 \times 10^{-4} \mathrm{~Pa}$. Periodicity was monitored by the low angle $\mathrm{x}$-ray diffraction method. The modulation period was kept at $12.8 \mathrm{~nm}$ (thicknesses of $\mathrm{As}_{2} \mathrm{~S}_{3}$ and Se films within a particular layer were 3.8 and $9 \mathrm{~nm}$, respectively) and the total thickness of the film was $0.86 \mu \mathrm{m}$. Se was deposited as the top and bottom layer of the multilayered film stack.

We used a pump probe technique to study the kinetics of photoinduced diffusion in our samples using the experimental setup that is described elsewhere. ${ }^{9}$ The sample was illuminated by a diode pumped solid state laser with wavelength of $532 \mathrm{~nm}$ (pump beam) and an intensity of $1 \mathrm{~W} / \mathrm{cm}^{2}$ at room temperature. During illumination, the transmission of the sample was probed using a white light source with wavelength ranging from 450 to $1000 \mathrm{~nm}$. The spectrometer had the capability to collect the entire optical spectrum in $2 \mathrm{~ms}$.
In the present experiments, the entire optical spectrum was collected in real time of $500 \mathrm{~ms} /$ spectrum. The pump beam was expanded using a beam expander to encompass an area that was larger than that of the probe beam, so that an area of uniform light intensity was examined. The transmission spectrum through the film was recorded before and during illumination.

To study the atomic mass transport and the different bonds formed during photoinduced diffusion, we used the XPS. The spectra were recorded at two states, i.e., unirradiated and irradiated states of the samples. Measurements were first made on the as prepared samples. The samples were then irradiated inside the XPS chamber with a He-Ne laser at different time intervals at the same spot where the XPS measurements were done. After irradiation, XPS spectra were again recorded at the irradiated area. This will allow us to make a clear correlation between the XPS data of as prepared and irradiated state of the sample. Since the time required to record highly resolved XPS spectra of any particular element, for example, As or Se, is much longer than the characteristic diffusion times, we irradiated the sample for a definite time and the measurements were carried out. The same spot was again irradiated and the same procedure was repeated. The XPS core level and valence band spectra were obtained with monochromatic $\mathrm{Al} K \alpha$ x rays $(1486.6 \mathrm{eV})$ at a vacuum of $10^{-9}$ Torr. The details of a particular XPS scan are pass energy at $40 \mathrm{eV}$, step size of $0.1 \mathrm{eV}$, and the data are averaged at three times. For insulators such as glasses, the charging effect can change the Binding Energy (BE) of the electrons from sample to sample. Hence the measurement of the absolute $\mathrm{BE}$ of electrons from a specified energy level is not reliable. The $\mathrm{C} 1 s$ line from either adventitious carbon or intentionally added graphite powder on the surface has been widely used for charge referencing. ${ }^{10,11}$ For this study, the adventitious carbon was used as a reference and the BE of the reference C $1 s$ line was set as $284.6 \mathrm{eV}$. For each sample, a calibration factor was calculated from the difference between the measured $\mathrm{C} 1 s \mathrm{BE}$ and the reference value of $284.6 \mathrm{eV}^{12}$ The original BE data were corrected according to the calibration factor.

\section{RESULTS}

Optical transmission spectra of nanolayered $\mathrm{Se} / \mathrm{As}_{2} \mathrm{~S}_{3}$ films are shown in Fig. 1 as a function of wavelength at different times of irradiation. The signal of the pump beam at $532 \mathrm{~nm}$ appears on the spectrum as a very high intensity line. Optical absorption edge was determined from the data of Fig. 1 based on the model of effective optical media. ${ }^{1,2}$ According to this model, narrow bandgap "well" layers determine the absorption and the contribution from the wide bandgap "barrier" layers is small. The effective optical bandgap of our samples was determined using the equation

$$
\alpha h \nu=B\left(h \nu-E_{g}\right)^{p},
$$

Where $\alpha, h, \nu, p, E_{g}$, and $B$ are absorption coefficient, Plank's constant, frequency, an integer, optical bandgap, and a constant (Tauc parameter), respectively. Equation (1) is valid for a number of amorphous materials in the spectral 


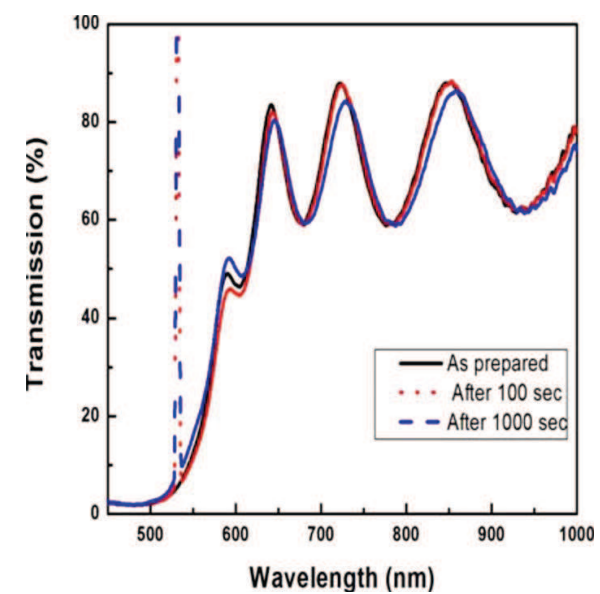

FIG. 1. (Color online) Transmission spectra of $\mathrm{Se} / \mathrm{As}_{2} \mathrm{~S}_{3}$.

region of large $\alpha\left(10^{4}<\alpha<10^{5}\right)$, i.e., Tauc region. ${ }^{2,3,13}$ In the special case where both the conduction and valence band edges have a parabolic shape, the value of $p$ is 2 . This quadratic dependence was observed in many chalcogenide glasses like $\mathrm{As}_{2} \mathrm{~S}_{3}, \mathrm{As}_{2} \mathrm{Se}_{3}, \mathrm{As}_{2} \mathrm{Te}_{3}$, etc., ${ }^{14}$ but not all chalcogenide glasses. The values of $p$ are 1 and 3 for $a$-Se and multicomponent chalcogenide glasses (e.g., Ge-As-Te-Si), respectively. ${ }^{14}$ For our $\mathrm{Se} / \mathrm{As}_{2} \mathrm{~S}_{3}$ nanolayered films, Se layers with lower bandgap $(2 \mathrm{eV})$ determine the optical absorption edge since the contribution from the wide bandgap $\mathrm{As}_{2} \mathrm{~S}_{3}(2.4 \mathrm{eV})$ is small. We fitted the optical absorption spectrum using Levenberg-Marquardt $\chi^{2}$ minimizing algorithm, which is one of the most widely used algorithms with least squares fitting. The best fit to Eq. (1) shows that the value of $p$ is equal to 1 . Earlier studies on $\mathrm{Se} / \mathrm{As}_{2} \mathrm{~S}_{3}$ multilayered samples [where the $\mathrm{Se}, \mathrm{As}_{2} \mathrm{~S}_{3}$ sublayer thickness ratio was very small $(0.3$ or less $)]$ reported the value of $p=2$. $^{2,3}$ We assume that the main reason for the quadratic energy dependence of $\alpha h \nu$ in those samples was the quantum confinement effect, which is different in the present samples in two ways. First, the Se sublayer thickness (less than $5 \mathrm{~nm}$ ) was lower compared to the $9 \mathrm{~nm}$ sublayer thickness of the present sample. Second, the $\mathrm{As}_{2} \mathrm{~S}_{3}$ sublayer thickness $(12 \mathrm{~nm}$ or above) was larger compared to the $3 \mathrm{~nm}$ of the present sample, which poses a stronger potential barrier to the well layers. Figure 2 shows that the calculated optical bandgap decreases initially, then starts increasing, ultimately saturating after $1000 \mathrm{~s}$ of laser exposure.

Constant $B$ in Eq. (1) includes information on the convolution of the valence band and conduction band states and on the matrix element of optical transitions, which reflects not only the relaxed $k$ selection rule but also the disorder induced spatial correlation of optical transitions between the valence band and conduction band. ${ }^{15}$ Moreover $B$ changes with the degree of disorder in the film. ${ }^{15}$ It also shows the same time dependence as the bandgap and suggests that initially the disorder in the film increases and then decreases (see Fig. 2).

We also measured the time evolution of the change in absorption coefficient $\Delta \alpha$ from the changes in transmission at five different wavelengths in the strong absorption part of

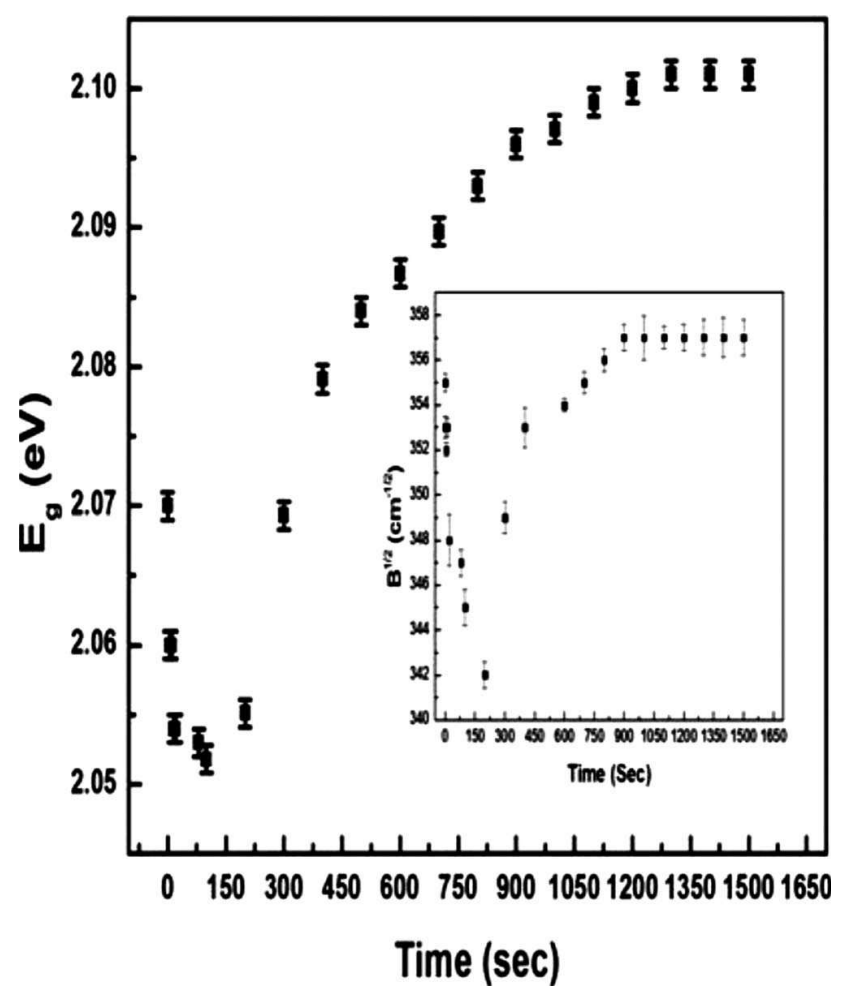

FIG. 2. Changes in the optical absorption edge during irradiation as a function of time. Inset: variation of Tauc parameter $\left(B^{1 / 2}\right)$ during irradiation.

the spectrum as shown in Fig. 3. Note that during illumination, $\Delta \alpha$ increases for first few seconds, saturates, and then starts decreasing.

The XPS results clearly show the atomic movements during photodiffusion. The BE of As atoms shifts to lower values upon irradiation, indicating the As-Se bond formation (Fig. 4). From the BE spectrum of Se, we again concluded the As-Se bond formation (Fig. 5). Since Se peaks are in the close vicinity of the $\mathrm{S}$ peaks and moreover the Se peak intensities are much higher than that of the S peak, we have not used the S peaks for further XPS analysis.

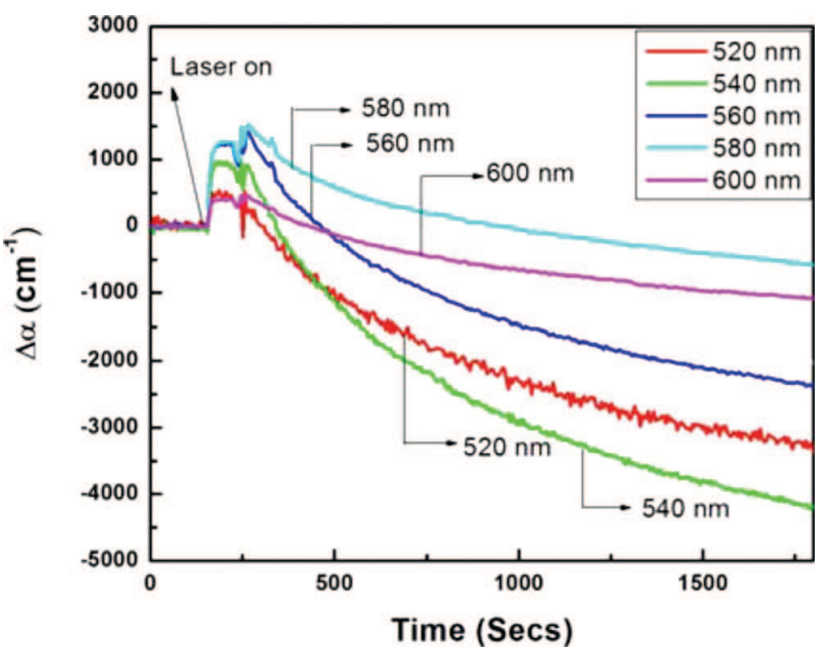

FIG. 3. (Color online) Time evolution of absorption coefficient with time at indicated wavelengths. The laser on state is indicated in the figure. 


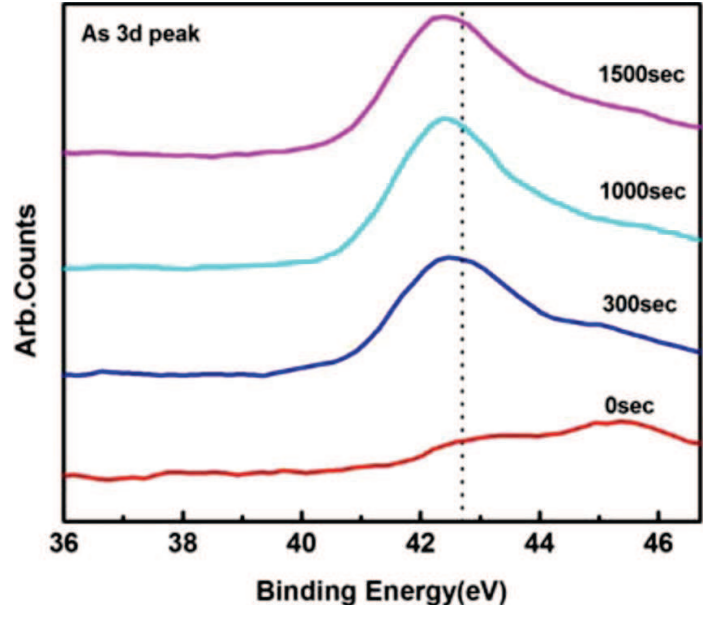

FIG. 4. (Color online) XPS As $3 d$ spectra of as prepared and irradiated (at different times) $\mathrm{Se} / \mathrm{As}_{2} \mathrm{~S}_{3}$ nanolayered films. Please note that there was no As $3 d$ peak for the as prepared sample. However after irradiating the sample, we can clearly see the signal from As $3 d$ peak.

\section{DISCUSSIONS}

From Fig. 1, it appears that photodarkening (decrease in bandgap or redshift) takes place in the irradiated region at first, gradually followed by photoinduced diffusion. We have expected only photobleaching effect during irradiation because of the following two reasons: (1) any photoinduced diffusion of Se into $\mathrm{As}_{2} \mathrm{~S}_{3}$ should increase the bandgap ${ }^{3}$ and (2) the decrease in Se thickness by photoinduced diffusion favors an increase in bandgap due to quantum size effects. ${ }^{1,2}$ The top of the valence band in this multilayered film stack is formed by the nonbonding orbitals of the Se atoms. Change in the position of $\mathrm{Se}$ atoms due to new bond formation causes a change in interaction between the lone pair electrons, which in turn changes the forbidden gap. ${ }^{16}$ During light irradiation, one of the Se lone pair electrons from the valence band gets excited into the conduction band, leaving behind the other in the lone pair orbital. Provided the distance between such excited atoms is close to the covalent

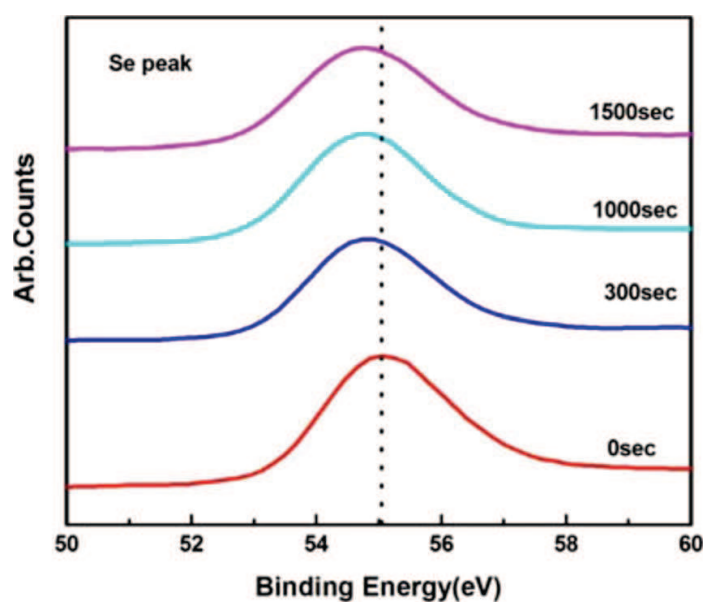

FIG. 5. (Color online) XPS Se $3 d$ spectra of as prepared and irradiated (at different times) $\mathrm{Se} / \mathrm{As}_{2} \mathrm{~S}_{3}$ nanolayered films. Please note that with irradiation the Se peaks shift to lower BE, which clearly indicating the formation of As-Se bond.

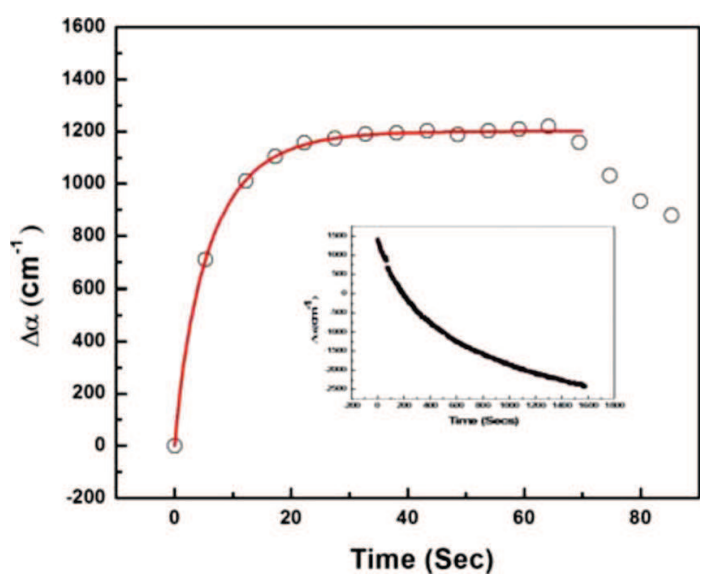

FIG. 6. (Color online) Hollow circles represent the rise in optical absorption edge during photodarkening and the solid line (red) shows the theoretical fit using Eq. (3). Inset: the solid squares show the decay of optical absorption edge during photoinduced diffusion and solid line (red) represents the theoretical fit to $E$.

bond length and the spins have opposite directions, additional covalent bonds can be dynamically formed between excited atoms, making some of the selenium atoms threefold coordinated. The formation of the dynamical bonds causes the displacement of nearest neighbor atoms slip away from equilibrium position, resulting in an increase in the total lone pair-lone pair interactions. It leads to the widening of the valence band, resulting in the reduction in bandgap (photodarkening). ${ }^{17}$

The rate of growth of the number $(N)$ of Se atoms can be written as ${ }^{18,19}$

$$
\frac{d N}{d t}=k_{p}\left(N_{T}-N\right)-k_{r} N
$$

where $N_{T}$ is the total number of atoms in the ground state which take part in this photon assisted site switching, $k_{p}$ is promotion rate given by $A t^{\beta-1}$, and $K_{r}$ is the recovery rate given by $B t^{\beta-1}$, where $A$ and $B$ are temperature and intensity dependent constants and $\beta$ is the dispersion parameter which is the same for both forward and backward reactions. $N$ can be written as $N=N_{s}\{1-\exp (-t / \tau)\}$, where $N_{s}=A N_{T} /(A+B)$ and $\tau=\{\beta /(A+B)\}^{1 / 2}$ are the saturated number of photodarkened sites and the effective reaction time, respectively. Then the effective increase in the absorption coefficient can be written as ${ }^{18}$

$$
\Delta \alpha=\Delta \alpha_{s}\left[1-\exp \left\{-(t / \tau)^{\beta}\right\}\right],
$$

where $t, \Delta \alpha$, and $\Delta \alpha_{s}$ are the time after the laser is switched on, change in absorption coefficient, and saturated value of $\Delta \alpha$, respectively. Equation (3) gives a reasonable fit to the experimental data (Fig. 6) and the values of $\Delta \alpha_{s}, \beta$, and $\tau$ are listed in Table. I.

We find that $\beta$ and $\tau$ are independent of wavelength, but $\Delta \alpha_{s}$ is wavelength dependent. The photodarkening has not been observed in homogenous film of Se at room temperature, but there are reports about its observation in Se at low temperatures. ${ }^{16}$ The main reason for the lack of photodarkening in Se at room temperature is mainly because the glass transition temperature of Se (Ref. 20) is just above room 
TABLE I. Fitting parameters obtained from fitting Eqs. (3) and (4) to the calculated rise and fall of the absorption coefficient at different wavelengths.

\begin{tabular}{ccccccc}
\hline \hline $\begin{array}{c}\lambda \\
(\mathrm{nm})\end{array}$ & $\begin{array}{c}\tau \\
(\mathrm{s})\end{array}$ & $\beta$ & $\begin{array}{c}\Delta \alpha_{s} \\
\left(\mathrm{~cm}^{-1}\right)\end{array}$ & $\begin{array}{c}\tau_{p} \\
(\mathrm{~s})\end{array}$ & $\beta_{p}$ & $\begin{array}{c}\Delta \alpha_{s p} \\
\left(\mathrm{~cm}^{-1}\right)\end{array}$ \\
\hline 520 & 4 & 0.9 & 518 & 928 & 0.7 & 4312 \\
540 & 6 & 0.9 & 1061 & 808 & 0.7 & 5505 \\
560 & 6 & 0.89 & 1201 & 731 & 0.7 & 3233 \\
580 & 6 & 0.89 & 1253 & 1128 & 0.65 & 1525 \\
600 & 6 & 0.9 & 400 & 1173 & 0.7 & 1760 \\
\hline \hline
\end{tabular}

temperature and the defects, which are responsible for photodarkening, anneal out at room temperature. ${ }^{4}$ However, when the Se layers of nanometer thickness are confined between the $\mathrm{As}_{2} \mathrm{~S}_{3}$ layers, the glass transition temperature $\left(T_{g}\right)$ shifts to higher values due to the quantum confinement effects, which provides stability to the defects responsible for photodarkening. There is continuing debate about whether the glass transition is a thermodynamic transition or a purely kinetic phenomenon. ${ }^{21}$ Adam and Hibbs ${ }^{22}$ introduced the concept of cooperative rearrangement of regions to unify these two views of glass transition. Donth ${ }^{23}$ estimated the size of such cooperatively rearranging regions to be of the order of $10 \AA$ for a number of glasses. When Se is confined between $\mathrm{As}_{2} \mathrm{~S}_{3}$ and the thickness of $\mathrm{Se}$ is comparable with the cooperative rearranging regions, it is possible to observe the quantum size effects on the glass transition temperature of Se. Under this condition, the dynamics of the interfacial regions can be slower than that of the rest of the system because of the interaction of interfacial molecules with the boundaries. $^{21,24}$ Depending on the relative strength of these effects in confined system and interfacial interactions, relaxation becomes quicker or slower, which may result in an increase or decrease in $T_{g}$. It was observed that the $T_{g}$ of Se shifts to higher temperature with decrease in Se thickness/ size in $\mathrm{Se} / \mathrm{As}_{2} \mathrm{Se}_{3}$ multilayers and $\mathrm{Se}$ confined in zeolites. $^{25,26}$ Moreover the experiments on holographic recording in $\mathrm{Se} / \mathrm{As}_{2} \mathrm{~S}_{3}$ samples also suggest the glass transition temperature shifts to higher temperatures. ${ }^{27}$ The observation of photodarkening in our multilayered film at room temperature provides an indirect proof of the shift of $T_{g}$ to higher temperature under confined conditions.

After the first few seconds, the number of photodarkened defects saturates, which produces local stress in the Se layer. These threefold coordinated Se atoms are highly unstable at room temperature. We assume that at this point, some of these Se atoms undergo bond switching with the $\mathrm{As}_{2} \mathrm{~S}_{3}$ layer containing normal twofold coordinated Se atoms. A combined effect of the removal of the threefold coordinated Se atoms and the new bond formation of Se atoms with $\mathrm{As}_{2} \mathrm{~S}_{3}$ results in an increase in the optical bandgap. These bond rearrangements continue as long as the material is exposed to light. The local bond changes and atomic motions tend to decrease the local strain energy, and the photodiffusion continues until the local stresses are released. The increase in bandgap causes a decrease in absorption coefficient. By using Eq. (2), this decay in absorption coefficient can be described by the equation

$$
\Delta \alpha=A\left(\exp \left\{-\left(t / \tau_{p}\right)^{\beta_{p}}\right\}\right)+\Delta \alpha_{p}
$$

where $t, \tau_{p}, \beta_{p}, \Delta \alpha_{p}$, and $A$ are the time after the absorption coefficient starts decaying, the effective reaction time constant, dispersion parameter $\left(0<\beta_{p}<1\right)$, the persistent value of absorption coefficient, and a temperature dependent parameter, respectively. Equation (4) gives a reasonable fit to the experimental data (see inset of Fig. 6) with the fitting parameters as listed in Table I. The reaction time $\tau_{p}$ increases with the decrease in wavelength, indicating that at lower wavelength the diffusion is more efficient. The main reason may be because at higher wavelengths only the lower bandgap layer will absorb the light, but at lower wavelengths both the layers will absorb the light. The dispersion parameter, $\beta_{p}$, is wavelength independent, but $\Delta \alpha_{p}$ is wavelength dependent.

$B^{1 / 2}$ is a very useful parameter to determine the degree of disorder during photoinduced diffusion (provided that the change in disorder during photodiffusion will change the valence and conduction band densities of states). Based on the experiments in $a-\mathrm{Ge}-\mathrm{H}, a-\mathrm{Si}-\mathrm{H}, a-\mathrm{SiN}$, and $a-\mathrm{SiN}$ based alloys, Zanatta and Chambouleyron ${ }^{15}$ showed that $B^{1 / 2}$ is sensitive to topological disorder only when electronic structural changes occur. The variation of $B^{1 / 2}$ as a function of time is shown in Fig. 2, which shows that the disorder increases at the time of photodarkening and decreases during photodiffusion. Kolobov et al. ${ }^{16}$ observed a light-induced increase in the average coordination number (by $\sim 5 \%$ ) and disorder in $a$-Se at $30 \mathrm{~K}$, while the bond length remained unchanged. This observation was ascribed to the local formation of additional dynamical threefold coordinated sites, which increased structural disorder. From the above discussion the photodarkening in our multilayered film appears to be due to the creation of threefold coordinated Se atoms. The creation of these Se defects increases the structural disorder, which in turn produces a decrease in the value of $B^{1 / 2}$. However, during photodiffusion the density of threefold coordinated Se atoms decreases, which reduces the structural disorder in the multilayered film. The reduction in threefold coordinated Se and the creation of new bonds with As and S result in a more chemically ordered state. As a consequence, the absorption edge becomes steeper and the magnitude of $B^{1 / 2}$ increases. Moreover the reduction in $\mathrm{Se}-\mathrm{Se}$ bonds also favors a high $B^{1 / 2}$ value. In the case of $a-\mathrm{Si} / a$-Ge alloys a change in $B^{1 / 2}$ has been found with decrease in $\mathrm{Ge}-\mathrm{Ge} / \mathrm{Si}-\mathrm{Si}$ bond density. ${ }^{28}$

The XPS analysis provided more insight into the atomic movements during photodiffusion. From Fig. 4, it was clear that there was no As peaks for the as prepared sample, since the top layer was formed by the Se atoms and hence almost all the signals were from that layer. After irradiating the sample for $300 \mathrm{~s}$, photoinduced diffusion takes place and the As atoms move toward the top surface, which contribute to a considerable XPS signal. When we closely look at the XPS signal at different times of irradiation, it was observed that the BEs of the As atoms are shifting toward the lower end with increase in irradiation time. The reported results showed that the $\mathrm{BE}$ of $\mathrm{As}$ in $\mathrm{As}_{2} \mathrm{~S}_{3}$ is $43 \mathrm{eV}^{3}$ The $\mathrm{BE}$ of As atom for irradiated sample for $300 \mathrm{~s}$ is at $42.7 \mathrm{eV}$ and the same for 
$1500 \mathrm{~s}$ is at $42.4 \mathrm{eV}$. From this we could make out that some of the As-S bonds are broken and As-Se bonds are created. Since Se's electronegativity is greater than As but less than $\mathrm{S}$, the BE of the As-Se bond will lie between the As-As (41 $\mathrm{eV})$ and As-S (43 eV) bonds. ${ }^{29}$ So it is evident that during photodiffusion As-Se bonds are formed. The earlier ${ }^{3}$ reported results showed that As-As bonds are present in the as prepared samples. These bonds are converted into As-S or As-Se bonds with light irradiation. Here they assumed that the phonon assisted As- $(\mathrm{S} / \mathrm{Se})$ bond formation takes place by using the lone pair electrons of the chalcogen atoms. However our XPS measurements at different times reveal that the As-Se bond formation takes place not only from the wrong As-As bonds but also by breaking some of the As-S bonds. Moreover in the former case, the top layer is formed from the $\mathrm{As}_{2} \mathrm{~S}_{3}$, one can get detailed information about the structural rearrangement of As atoms in the $\mathrm{As}_{2} \mathrm{~S}_{3}$ layer. However in our case, the top layer is formed of Se atoms, and this layer is sufficiently thick that there was no As signal. Once we irradiate the sample for $300 \mathrm{~s}$ and measure the XPS, the XPS signal of the As atoms comes from the solid solution of $\mathrm{Se}_{x}\left(\mathrm{As}_{2} \mathrm{~S}_{3}\right)_{1-x}$. Again we irradiate this sample; some of the As atoms bonded with $\mathrm{S}$ atoms are broken and the As-Se bonds are created. This can be easily visualized by looking at the lowering of the BE of As atoms with higher irradiation times. Our XPS measurements do not give any idea about how the diffusion takes place but give the detailed information about the bond rearrangement of As atoms with $\mathrm{S}$ and Se.

If we compare the $\mathrm{Se} 3 d \mathrm{BE}$ of the as prepared and irradiated samples, we can infer the following. It is clear that during photodiffusion Se is forming bonds with As. BE of Se for the as prepared sample is at $55 \mathrm{eV}$ and the $\mathrm{BE}$ of $\mathrm{Se}$ is decreasing with increase in irradiation time. The $\mathrm{BE}$ at $55 \mathrm{eV}$ is due to the $\mathrm{Se}-\mathrm{Se}$ bond. When we irradiate the sample, photoinduced diffusion takes place and some of the Se-Se bonds are converted into As-Se bonds. Since the electronegativity of Se is greater than the electronegativity of As, the $\mathrm{BE}$ of Se shifts to lower values. The As-Se bond formation is also clearly visible when we analyzed the As bonds. We also assume that $\mathrm{Se}$ is forming bonds with $\mathrm{S}$, but the number of Se atoms forming bonds with $\mathrm{S}$ atoms may be very less and that may be one of the reasons for not seeing the S-Se bonds in our experiments.

The previous reported results showed that photoinduced diffusion takes place through the interaction of $\mathrm{Se}_{2}^{0}$ atoms with wrong $\mathrm{S}-\mathrm{S}$ and As-As bonds. ${ }^{3}$ The present experimental results show that these $\mathrm{Se}_{2}^{0}$ sites are first converted into $\mathrm{Se}_{3}^{0}$ sites, which then react with $\mathrm{As}-\mathrm{S}, \mathrm{As}-\mathrm{As}$, and $\mathrm{S}-\mathrm{S}$ bonds. From the in situ optical absorption measurements, we also show that there is an induction time before the diffusion and at this time the $\mathrm{Se}$ atoms are rearranging themselves. Nevertheless our experimental results clearly show that some of the As-S bonds are converted into As-Se bonds. Since it is energetically difficult to break an As-S bond to form an As-Se bond, we assume that the new bond formations are taking place by the bond rearrangement mechanism. Our optical measurements clearly show the bond rearrangements by which $\mathrm{Se}_{2}^{0}$ atoms are first converted into $\mathrm{Se}_{3}^{0}$ sites. These highly unstable $\mathrm{Se}_{3}^{0}$ sites react with $\mathrm{As}-\mathrm{S}$, As-As bonds forming As-Se bonds.

\section{CONCLUSIONS}

The in situ optical absorption measurements reveal that the photodarkening in amorphous nanolayered $\mathrm{Se} / \mathrm{As}_{2} \mathrm{~S}_{3}$ follows photoinduced diffusion. Photodarkening appears to be due to the structural rearrangements in the Se layer upon irradiation, which causes a displacement of neighboring atoms from equilibrium positions. It results in an increase in the total lone pair-lone pair interactions, which leads to the widening of valence band and hence photodarkening. The movement of atoms from their equilibrium position increases the structural disorder, as indicated from the analysis of Tauc parameter. Some of the threefold coordinated Se atoms react with the $\mathrm{As}_{2} \mathrm{~S}_{3}$ layer producing photoinduced diffusion.

The XPS results clearly show the atomic movements during photoinduced diffusion. From our experiments we are able to show that some of the As-S bonds are converted into As-Se bonds. There is a considerable decrease in As-S bond followed by an increase in the As-Se and S-Se bonds. Since it is energetically difficult to break an As-S bond to form an As-Se bond, we assume that the new bond formations are taking place by the bond rearrangement mechanism. Our optical measurements clearly show the bond rearrangements by which $\mathrm{Se}_{2}^{0}$ atoms are first converted into $\mathrm{Se}_{3}^{0}$ sites. These highly unstable $\mathrm{Se}_{3}^{0}$ sites react with As-S, As-As bonds forming As-Se bonds.

\section{ACKNOWLEDGMENTS}

The authors thank the bilateral Indo-Hungarian research project, NSF's International Materials Institute for New Functionality in Glass (Grant No. DMR-0409588), and CSIR-SRF Grants for supporting this multinational collaboration. The authors thank Bill Heffner, Ashtosh Ganjoo, and $\mathrm{K}$. Antoine of Lehigh University for their help and valuable suggestions.

${ }^{1}$ M. Malyovanik, S. Ivan, A. Csik, G. A. Langer, D. L. Beke, and S. Kokenyesi, J. Appl. Phys. 93, 139 (2003).

${ }^{2}$ K. V. Adarsh, K. S. Sangunni, S. Kokenyesi, S. Ivan, and M. Shipljak, J. Appl. Phys. 97, 044314 (2005).

${ }^{3}$ K. V. Adarsh, K. S. Sangunni, T. Shripathi, S. Kokenyesi, and M. Shipljak, J. Appl. Phys. 99, 094301 (2006).

${ }^{4}$ K. Shimakawa, A. V. Kolobov, and S. R. Elliot, Adv. Phys. 44, 475 (1995).

${ }^{5}$ A. V. Kolobov and S. R. Elliot, Adv. Phys. 40, 625 (1991).

${ }^{6}$ A. Kikineshi, V. Paylok, I. A. Szabo, M. Shipljak, I. Ivan, and D. L. Beke, J. Non-Cryst. Solids 326-327, 484 (2003).

${ }^{7}$ P. I. K. Onorato, M. N. Alexander, C. W. Struck, G. W. Tasker, and D. R. Uhlmann, J. Am. Ceram. Soc. 68, C148 (1985).

${ }^{8}$ J. Heo, J. S. Sanghera, and J. D. Mackenzie, J. Non-Cryst. Solids 101, 23 (1988).

${ }^{9}$ K. Antoine, H. Jain, and M. Vlcek, J. Non-Cryst. Solids 352, 595 (2006).

${ }^{10}$ J. F. Moulder, W. F. Sticker, P. E. Sobol, and K. D. Bomben, Hand Book of X-Ray Photoelectron Spectroscopy (Perkin Elmer, Eden Pairie, MN, 1992).

${ }^{11}$ W. J. Stec, W. E. Morgan, R. G. Albridge, and J. R. Van Wazer, Inorg. Chem. 11, 219 (1972).

${ }^{12}$ T. L. Barr and S. Seal, J. Vac. Sci. Technol. A 13, 1239 (1995).

${ }^{13}$ R. Bindemann and O. Paetzold, Phys. Status Solidi B 160, K183 (1990).

${ }^{14}$ S. R. Elliot, Materials Science and Technology: A Comprehensive Treatment (Wiley, New York, 1996), pp. 19 and 375. 
${ }^{15}$ A. R. Zanatta and I. Chambouleyron, Phys. Rev. B 53, 3833 (1996).

${ }^{16}$ A. V. Kolobov, H. Oyanagi, K. Tanaka, and K. Tanaka, Phys. Rev. B 55, 726 (1997).

${ }^{17}$ K. Shimakawa, N. Yoshida, A. Ganjoo, Y. Kuzukawa, and J. Singh, Philos. Mag. Lett. 77, 153 (1998).

${ }^{18}$ A. Ganjoo, K. Shimakawa, H. Kamiya, E. A. Davis, and J. Singh, Phys. Rev. B 62, R14601 (2000).

${ }^{19}$ A. Ganjoo and H. Jain, Phys. Rev. B 74, 024201 (2006).

${ }^{20}$ Z. U. Borisova, Glassy Semiconductors (Plenum, New York, 1981), Chap. 2, p. 39.

${ }^{21}$ J. A. Forrest, K. Dalmonki-Veess, J. R. Stevens, and J. R. Dutcher, Phys. Rev. Lett. 77, 2002 (1996).
${ }^{22}$ G. Adam and J. H. Hibbs, J. Chem. Phys. 55, 241 (1965).

${ }^{23}$ E. Donth, J. Non-Cryst. Solids 53, 325 (1982).

${ }^{24}$ B. Jerome and J. Commandeur, Nature (London) 386, 589 (1997).

${ }^{25}$ E. Maruyama, Jpn. J. Appl. Phys., Part 1 21, 213 (1982).

${ }^{26}$ K. Matsuishi, K.-i. Nogi, H. Ogura, S. Onari, and T. Arai, J. Non-Cryst. Solids 227-230, 799 (1998).

${ }^{27}$ V. Palyok, A. Mishak, I. Szabo, D. L. Beke, and A. Kikineshi, Appl. Phys. A: Mater. Sci. Process. 68, 489 (1999).

${ }^{28}$ J. Robertson, Philos. Mag. B 69, 307 (1994).

${ }^{29}$ CRC Handbook of Chemistry and Physics, 78th ed., edited by D. R. Lide (CRC, Boca Raton, FL, 1997). 